Research Article

\title{
Fixed Regimen Treatment in Unselected Naïve Patients Cohort with Neovascular Age-Related Macular Degeneration
}

\author{
Ricardo P. Casaroli-Marano $\mathbb{D}^{1,},{ }^{1,2}$ Carolina Bernal-Morales $\mathbb{D}^{1},{ }^{1}$ Lillian Chamorro-López, ${ }^{1}$ \\ Marina Dotti-Boada, ${ }^{1}$ Juan P. Figueroa-Vercellino, ${ }^{1}$ and Socorro Alforja ${ }^{1}$ \\ ${ }^{1}$ Service of Ophthalmology, Hospital Clinic de Barcelona, Barcelona, Spain \\ ${ }^{2}$ Department of Surgery, School of Medicine, University of Barcelona, Barcelona, Spain
}

Correspondence should be addressed to Ricardo P. Casaroli-Marano; rcasaroli@ub.edu

Received 18 August 2020; Revised 31 October 2020; Accepted 6 November 2020; Published 21 November 2020

Academic Editor: Ciro Costagliola

Copyright (c) 2020 Ricardo P. Casaroli-Marano et al. This is an open access article distributed under the Creative Commons Attribution License, which permits unrestricted use, distribution, and reproduction in any medium, provided the original work is properly cited.

\begin{abstract}
The current treatment of neovascular age-related macular degeneration (nAMD) generates an excessive care pressure in the public health system. The search for a satisfactory treatment regimen, whose anatomical and functional stability may be achieved, is a challenge and a goal to be reached. We analyzed the outcomes in a patient cohort under fixed regimen treatment with intravitreal aflibercept (IVA). A retrospective study, with at least 1-year follow-up, in consecutive treated unselected naïve patients was carried out. Standard protocol was performed and evaluated at baseline, month 4 (after loading dose, LD), and month 12 (after fixed bimonthly regimen). One hundred six patients (123 eyes) aged $80.3 \pm 7.7$ years were included, receiving $6.8 \pm 0.7$ IVA. Visual acuity gain after LD was $5.5 \pm 12.0$ letters $(p<0.0001)$. At month 12,23 eyes $(18.7 \%)$ gained $\geq 15$ letters and $58(47.1 \%)$ had bestcorrected visual acuity $\geq 20 / 40$ (70 letters). The improvement in visual acuity was lower in patients with polypoidal choroidal vasculopathy $(+4.9 \pm 18.1$ letters; $p=0.2544)$ and somewhat higher in patients with retinal angiomatous proliferation $(+5.4 \pm 12.3$ letters; $p=0.0373)$. Dry macula was present in only $9.8 \%$ of cohort at baseline vs. $69.7 \%$ at month $12(p<0.0001)$. Atrophy was the most observed complication and related to the elderly patients. The average of follow-up visits was $3 \pm 0.5$. Functional and anatomical improvement were observed with IVA in a fixed bimonthly regimen treatment after LD, with results maintained up to one year with a good compliance. The fixed bimonthly regimen optimized patient management and logistic issues.
\end{abstract}

\section{Introduction}

Age-related macular degeneration (AMD) is the leading cause of visual impairment and blindness in developed countries [1-3]. Neovascular AMD (nAMD) involves events of choroidal neovascularization $(\mathrm{CNV})$ with retinal oedema, hemorrhages, and functional impairment, which accounts for the majority of cases of severe visual loss among AMD patients [4]. The prevalence of nAMD increases with advancing age, rising from $1.4 \%$ at the age of 70 years to $20 \%$ at the age of 90 years $[5,6]$. In addition, studies on the estimates of those affected today and for the next years find that both the prevalence and incidence of AMD are constantly increasing in the elderly European population, with a projected increase of $15 \%$ in its prevalence and $75 \%$ in its incidence [7].
Although several potential therapeutic targets exist, the main pharmacological treatment for nAMD is blocking the action of vascular endothelial growth factor (VEGF). Experience accumulated during everyday clinical practice with intravitreal therapy with anti-VEGF confirmed that real-life outcomes are generally less impressive than those reported in clinical trials [8-10]. Delay of initial diagnosis and prompt treatment and/or discontinuous follow-up in routine clinical care are, among others, main contributors to these poorer results [8-11]. The burden of treatment has proven to be an important reason for anti-VEGF discontinuation, especially in the elderly, as early as within the first year of treatment [11]. Furthermore, logistic issues and overpressing healthcare contribute to worse outcomes and to difficult patient's management. 
Aflibercept has been shown to bind VEGF-A, VEGF-B, and placental growth factor (PGF) with higher affinity than the other currently available anti-VEGFs, presenting a longer intravitreal half-life, which may translate into less need for injection and follow-up controls [12]. These contribute to reducing care pressing in public health system environment. In this sense, pivotal studies showed that intravitreal aflibercept administered in a fixed bimonthly regimen provided satisfactory improvement in visual acuity (VA) after 52 weeks of treatment [13]. Evidence in the clinical setting indicating that the functional results obtained are more modest than those observed in the pivotal clinical trials $[8,10,14-20]$. Patients included in these studies vary greatly with respect to sociodemographic and disease characteristics because of the differences in the time of diagnosis or in local prescription recommendations. Changes in recent years with respect to the variants included in this macular disease are also likely to have an influence. As a result, evidence provided by these studies may not be representative of other real-life scenarios. Further, according to the standardized guidelines issued at the time, variants such as polypoidal choroidal vasculopathy (PCV) and retinal angiomatous proliferation (RAP) are treated with similar approach as nAMD [8].

Under this context, we have retrospectively analyzed the functional and structural outcomes achieved in an nAMD naïve-patient cohort who were treated with IVA for one year using a fixed bimonthly regimen. Our aim was to evaluate its effectiveness and optimize patient's assessment in daily clinical practice in public specialized health care. We have analyzed the general results of our cohort, and a subanalysis study was conducted with the aim of verifying our visual and structural results in a real-world setting.

\section{Methods}

This was a retrospective, single-centre, observational study conducted in the service of ophthalmology of the Hospital Clínic de Barcelona (Barcelona, Spain), a community hospital that is part of the Catalan Public Hospital Network. The local Research Ethics Committee gave its approval (HCB/ 2018/1051) for the audit and retrospective evaluation of the electronic clinical history files, which was conducted according to the Declaration of Helsinki guidelines and Spanish data protection law (Ley Orgánica 05/2018). Patients provided written informed consent before starting intravitreal treatment.

2.1. Inclusion and Exclusion Criteria. Data from consecutive naïve patients with nAMD, including PCV or RAP [8], who received intravitreal aflibercept (IVA) treatment with at least one-year follow-up (visit at month 12 or 13) was extracted from the electronic medical records (SAP) used in our institution. According to our protocol, all patients with $\mathrm{nAMD}$ with no specific contraindications for IVA are eligible for this treatment, without any visual or anatomic restriction. All patients had received 3 consecutive monthly injections (load dose, LD) followed by continuous bimonthly fixed-interval dosing injections until completing one year ( month 11). Deviations from the 4 - or 8 -week intervals were not collected. Some patients may have been referred from other centres early during the LD period. These previous doses and clinical data were also taken into consideration in this study.

2.2. Procedures. In accordance with our standard normalized protocol, nAMD was diagnosed and classified on eye fundus examination and confirmed by spectral domain(SD-; AngioPlex ${ }^{\mathrm{TM}}$, Carl Zeiss Meditec) or swept source(SS-; Triton ${ }^{\mathrm{TM}}$ DRI, Topcon Inc.) optical coherence tomography (OCT). This protocol only allows other imaging modalities such as fluorescent angiography (FA) and indocyanine green angiography (ICGA) during follow-up in patients not responsive to treatment. Volumetric high-resolution OCT scans were acquired over a $6 \times 6 \mathrm{~mm}$ field centred on the fovea to fully capture the lesion. OCT scans satisfied consensus criteria for retinal OCT quality assessment (OSCAR-IB) [21]. A single observer assessed macular morphology. Best-corrected visual acuity (BCVA) was evaluated at baseline using Early Treatment Diabetic Retinopathy Study (ETDRS) letters at a distance of 4 meters.

BCVA- and OCT-based outcomes assessed in this study are those at baseline, months 3-4 (after LD) and month 12 or 13 (after the 7th injection at month 11, at the end of the first year). Functional variables considered were mean change in BCVA, the number of eyes gaining $\geq 15$ or losing $\leq 5$ letters and achieving BCVA $\geq 20 / 40$ (70 letters). The structural variables analyzed included the central subfoveal macular thickness (CMT) - defined as the macular average thickness in the central $1 \mathrm{~mm}$ of the ETDRS grid-the average macular thickness (AMT) - defined as the mean thickness for each of the nine sectors of the entire $6 \mathrm{~mm}$ ETDRS grid-and the presence of intraretinal fluid (IRF), intraretinal cysts, subretinal fluid (SRF), and pigment epithelial detachment (PED), as assessed by OCT. The presence of hemorrhage, as well as retinal pigment epithelium (RPE) atrophy and fibrosis, was detected by biomicroscopy macular examination. The percentage of eyes with dry macula, defined as absence of IRF or cysts, and SRF fluid on OCT was also determined. BCVA gain in the different nAMD subtypes was assessed in an exploratory analysis given the small number of patients with PCV or RAP expected. Subanalysis by age group was also performed, taking into account a normal distribution of the cohort and the balanced differences between the different groups as follows: (a) patients $\leq 77$ and $>77$ years; (b) patients $\leq 80$ and $>80$ years; (c) patients $\leq 83$ and $>83$ years; (d) patients $\leq 85$ and $>85$ years. Safety variables included injection-related, ocular-related, and systemic adverse events.

2.3. Statistical Analysis. Patient and disease characteristics are described as continuous variables and expressed as mean (standard deviation [SD])/median (first and third quartiles [Q1; Q3]) and compared by the Mann-Whitney $U$ test or the $t$-test/ANOVA. Categorical variables are expressed as $n(\%)$. The evolution of variables over the course of the study period (baseline, months 4-5, and month 12) was analyzed using the 
paired $t$-test for continuous variables and Fisher's exact test for categorical variables. Missing observations were not imputed. There was no need to carry forward last observation. The SAS 9.4 statistical package was used. All $p$ values were two-sided; $p$ value $<0.05$ was considered statistically significant.

\section{Results}

A total of 106 patients (123 eyes) were included in the study. Mean age was $80.3 \pm 7.7$ years (range $58-98$ ) and 62 (58.5\%) were men. The nAMD was bilateral in 17 patients and 18 $(14.6 \%)$ eyes belonged to patients aged $\geq 90$ years. Nineteen $(15.4 \%)$ and $20(16.3 \%)$ eyes presented signs of PCV and RAP on SS- or SD-OCT $[22,23]$. The mean number of clinic visits was $3.0 \pm 0.5$. The mean number of IVA was $6.8 \pm 0.7$ (median: 7). Eleven (8.9\%), 69 (56.1\%), and 42 (34.1\%) eyes had received 8,7 , and 6 IVA, respectively. One $(0.8 \%)$ eye received only 3 injections, and treatment was stopped after LD due to the presence of an extensive subretinal fibrosis with very poor baseline BCVA $(<20 / 800 ; 4$ letters), while continuing in the fellow eye.

3.1. Functional Outcomes. Mean BCVA at baseline was 20/ 60 (58.7 \pm 17.1 letters). Thirteen (10.6\%) eyes had baseline BCVA $<20 / 300$ (25 letters): five eyes with 20/400 (20 letters) and 4 eyes with 20/800 (4 letters). After the LD, BCVA increased to $5.5 \pm 12.0$ letters $(p<0.0001)$, remaining stable up to month $12(p=0.5033)$. Mean BCVA at month 12 was $20 / 50$ (63.5 \pm 15.3 letters), with a mean change from baseline of $+4.9 \pm 14.1$ letters $(p=0.0002)$ (Figure 1$)$. Twenty-three eyes (18.7\%) gained $\geq 15$ letters after one year. In $33(26.8 \%)$ eyes, BCVA did not change from baseline (Table 1). The percentage of eyes presenting BCVA $\geq 20 / 40$ (70 letters) increased from $30.1 \%$ at baseline to $47.2 \%$ after $\mathrm{LD}$ $(p=0.0086)$ and remained stable $(46.3 \%)$ up to one year $(p<0.0001$ vs. baseline).

3.2. BCVA Evolution according to nAMD Subtype. Mean baseline BCVA was $54.5 \pm 18.9,56.3 \pm 23.0$, and $60.2 \pm 15.0$ letters in patients with RAP, PCV, and nAMD, respectively. BCVA gain after the LD was $7.7 \pm 9.4$ letters ( $p=0.0004$ vs. baseline) in patients with RAP and $4.8 \pm 11.9$ letters ( $p=0.0017$ vs. baseline) in patients with nAMD. BCVA remained stable up to month 12 in both cases, with an overall BCVA gain vs. baseline of $5.4 \pm 12.3(p=0.0373)$ and $4.8 \pm 14.1$ letters $(p=0.0024)$, for RAP and nAMD, respectively. In eyes with PCV, BCVA gain after LD was $6.3 \pm 14.7$ letters ( $p=0.0775$ vs. baseline) and remained stable up to one year $(4.9 \pm 18.1$ letters vs. baseline; $p=0.2544)$ (Figure 1).

3.3. Structural Outcomes. Mean CMT at baseline was $325.2 \pm 104.1 \mu \mathrm{m}$. After the LD, CMT decreased $79.1 \pm 94.5 \mu \mathrm{m}(p<0.0001)$. After one year, mean CMT was $240.7 \pm 56.2 \mu \mathrm{m}$, with a mean difference vs. baseline of $-84.5 \pm 97.6 \mu \mathrm{m}(p=0.0002)$ (Figure 1). Mean AMT at baseline was $287.9 \pm 42.6 \mu \mathrm{m}$, decreasing $28.3 \pm 36.2 \mu \mathrm{m}$ $(p<0.0001)$ after the LD and $3.9 \pm 16.8 \mu \mathrm{m}$ after one year $(p=0.0131)$. Mean AMT after one year was $255.7 \pm 27.3 \mu \mathrm{m}$, with a difference vs. baseline of $-32.2 \pm 40.0 \mu \mathrm{m}(p<0.0001)$ (Figure 1). RAP and PCV presented similar CMT pattern of improvement (Figure 1). Dry macula was present in only $9.8 \%$ of cohort at baseline vs. $69.7 \%$ at month 12 $(p<0.0001)$, with similar significant trend for nAMD subtypes (Figure 1). Changes in percentages of eyes with SRF-IRF, macular hemorrhage, and RPE atrophy were significant after LD (Figure 2 and Table 2).

3.4. Outcomes according to Age Groups. For this subanalysis, age groups have been considered taking into account a normal distribution of the cohort and the balanced differences between the different groups as follows: (a) patients $\leq 77$ years ( $n=43$ eyes); (b) patients $\leq 80$ years ( $n=59$ eyes); (c) patients $\leq 83$ years ( $n=79$ eyes); (d) patients $\leq 85$ years ( $n=89$ eyes); and (e) patients $>85$ years $(n=34$ eyes).

Mean changes in BCVA at final follow-up and at the baseline were compared (Figure 3 and Table 3 ). The eyes belonging to the older subgroups ( $>85$ years old), although they presented gains in the BCVA means, were not significant (Figure 3 and Table 3). Interestingly, gain of BCVA after the LD was $-4.6(p=0.06)$ and $-7.1(p=0.005)$ for the older subgroups, $>83$ and $>85$ years, respectively. Although there were not significant differences, the older subgroups tended to improve less BCVA on average at 12 months (Table 3). The decrease in the CMT and AMT means after the LD was significant for all subgroups remained stable until month 12 (not shown).

The presence of SRF-IRF and retinal hemorrhages has decreased significantly at the end of the study period (Figure 4). RPE atrophy was more frequent in the subgroup of patients with more than 85 years at the baseline $(24 \%$; $p<0.001)$. Although the presence of RPE atrophy increased significantly in all subgroups at 12 months (about 25\%; $p<0.005)$, this condition was more evident in the subgroup of patients older than 85 years $(56 \% ; p<0.001)$ (Figure 4$)$.

3.5. Safety. In our cohort, IVA was well tolerated. Severe adverse events were not observed, and mild adverse events were in line with its safety profile. Fourteen (11.4\%) eyes required emergency care because of post-IVA floaters, superficial punctate keratopathy, and traumatic hyposphagma due to intraocular injection.

\section{Discussion}

The results of our study support the effectiveness and safety of aflibercept, in a fixed regimen, for treatment-naïve patients with nAMD in clinical daily practice in a real-life scenario. The functional and structural benefits achieved confirm those of the two large pivotal clinical trials [13], as well as those others observational studies in clinical practice [14-20]. However, visual outcomes were lower than those reported previously [13], which may be explained by sociodemographic and disease-related differences in the 


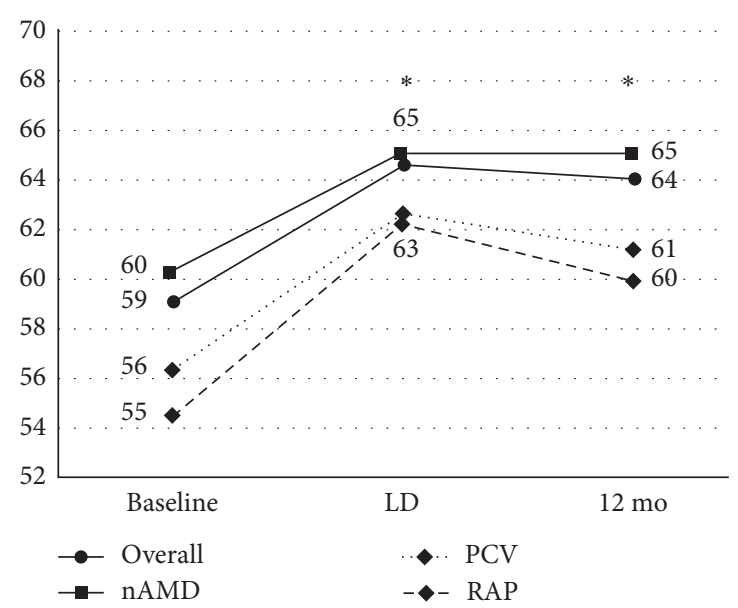

(a)

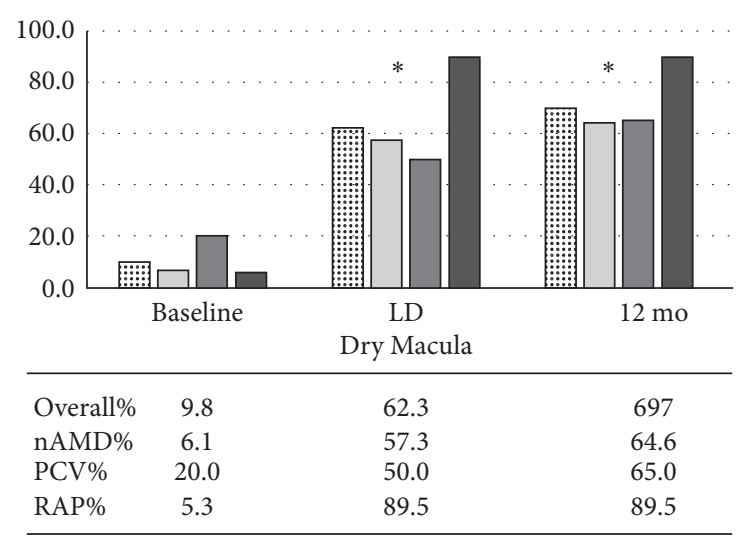

(c)

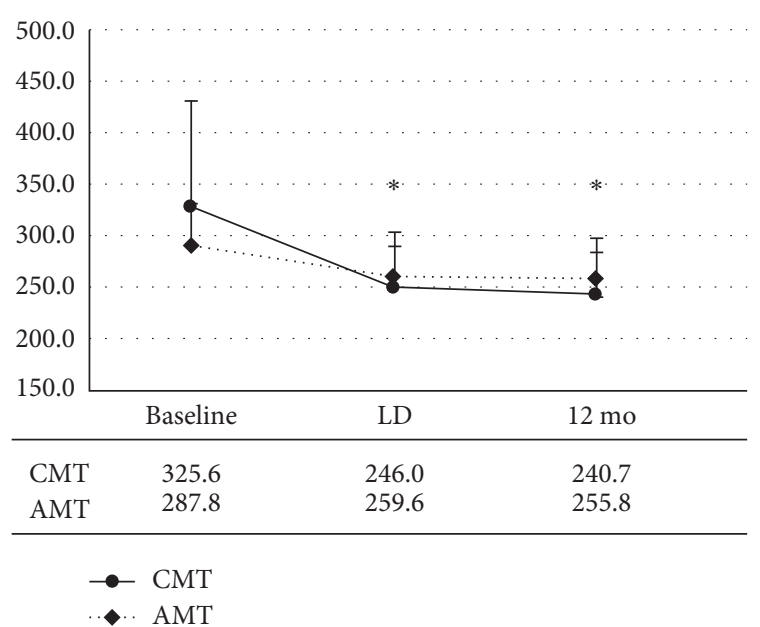

(b)

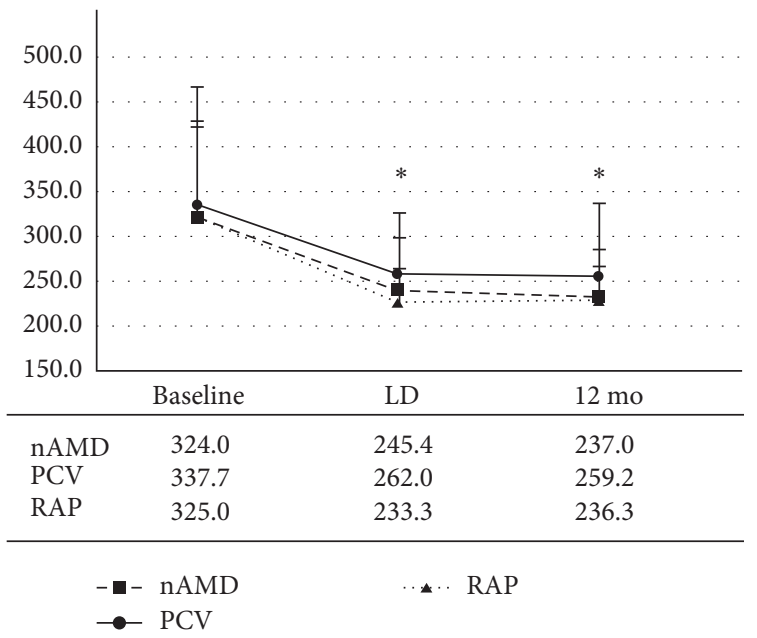

(d)

FIgure 1: Changes in BCVA (ETDRS letters), CMT $(\mu \mathrm{m})$, and AMT $(\mu \mathrm{m})$ and percentage of dry macula during the study period. (a) Significant gain $\left({ }^{*}\right)$ in mean BCVA was observed in overall $(p<0.0001)$, nAMD $(p=0.0004)$, and RAP $(p=0.0017)$ after the loading dose $(\mathrm{LD})$ and remained stable up to one year $(12 \mathrm{mo})$. BCVA changes in PCV were not significant. (b) Significant decrease $\left(^{*}\right)$ in mean CMT $(p<0.0001)$ and AMT $(p<0.0001)$ was observed after the LD and was maintained until month 12. (d) Significant $\left({ }^{*}\right)$ CMT improvement was also achieved for nAMD $(p<0.0001)$, PCV $(p<0.0001)$, and RAP $(p<0.0001)$. (c) The percentage of eyes with dry macula was significant $\left({ }^{*}\right)$ after the $\operatorname{LD}(p<0.0001)$ and also at 12 months $(p<0.0001)$.

populations included [18]. The same applies to the differences observed in VA gain with respect to other studies conducted with aflibercept in clinical practice, which at least in part are the result of the consideration for eligibility criteria for treatment according to local recommendations or protocols.

Contrary to the abovementioned studies, in accordance with everyday clinical practice in our setting, the only exclusion criteria in our study were those listed in the local summary product characteristics, with no restrictions with respect to baseline BCVA or the presence of structural damage to the fovea, or local or systemic comorbidities. These differences are likely to have had an effect on the visual outcomes achieved. Our study is also among the few to include patients with RAP and PCV, which has shown to be associated with differences in VA gain $[8,20,21]$. As
TABLE 1: Best-corrected visual acuity (number of letters) in overall cohort, after loading dose (LD) and at final follow-up period (12 mo).

\begin{tabular}{lccc}
\hline & Letters & LD & 12 mo \\
& & $n(\%)$ & $n(\%)$ \\
\hline Gain & 5 to 9 & $21(17.1)$ & $20(16.3)$ \\
& 10 to 19 & $26(21.1)$ & $28(22.8)$ \\
Equal & 020 & $10(8.1)$ & $12(9.7)$ \\
Loss & 0 to 4 & $47(38.3)$ & $33(26.8)$ \\
& 1 to 4 & $3(2.4)$ & $6(4.9)$ \\
& 5 to 9 & $8(6.5)$ & $10(8.1)$ \\
& $\geq 10$ & $8(6.5)$ & $14(11.4)$ \\
\hline
\end{tabular}

Letters: number of letters ETDRS; $n$ (\%): number of eyes (percentage); LD: loading dose; 12 mo: final follow-up.

expected, eyes with RAP benefited from greater VA gain (5.4 letters) compared to those with nAMD (4.8 letters). On the contrary, although eyes with PCV had an overall visual gain 


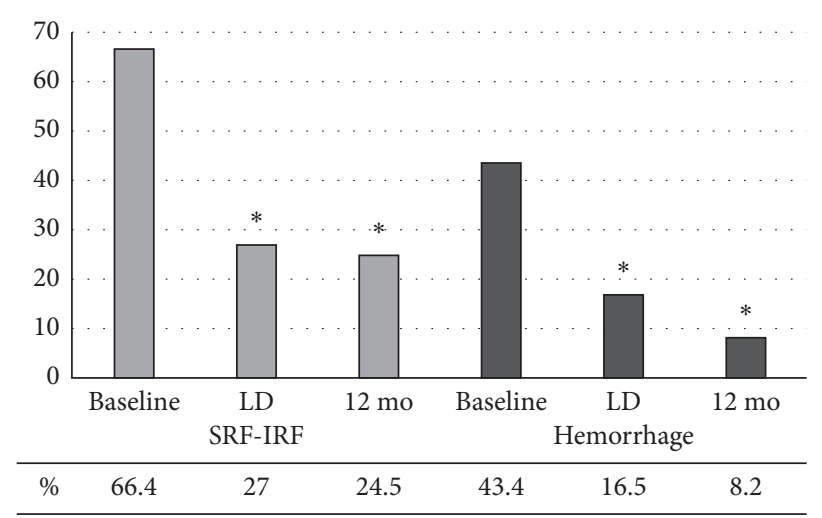

(a)

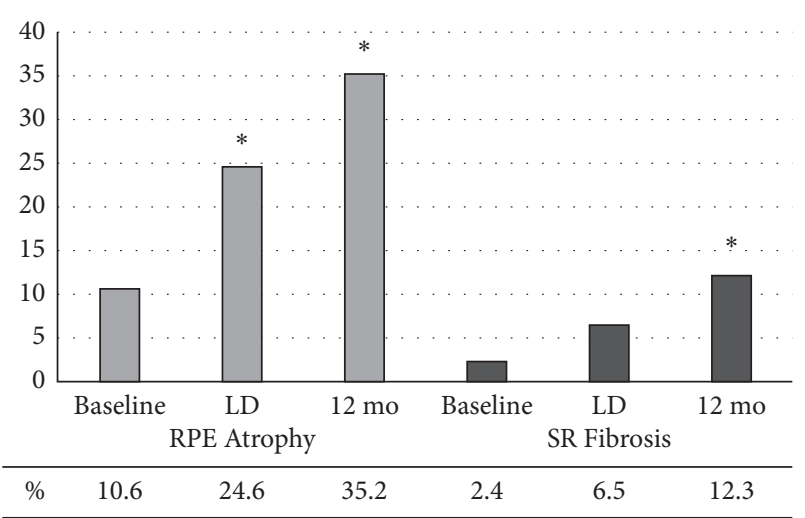

(b)

FIgURE 2: Presence of retinal findings during the period of study. (a) Changes in the percentage of eyes with subretinal fluid (SFR), intraretinal fluid (IRF), and hemorrhages, at baseline, after the loading dose (LD), and at 12 months (12 mo), were significant $\left({ }^{*}\right)$. (b) Changes in the percentage of eyes presenting retinal pigment epithelium (RPE) atrophy and subretinal (SR) fibrosis, at baseline, after the LD, and at $12 \mathrm{mo}$, were also significant $\left({ }^{*}\right)$.

TABLE 2: Prevalence of eyes with hemorrhage, RPE atrophy, subretinal fibrosis, and dry macula at baseline, after the loading dose (LD), and at final follow-up period $(12 \mathrm{mo})$.

\begin{tabular}{lccccc}
\hline & Baseline $n(\%)$ & LD $n(\%)$ & $p \mathrm{a}$ & 12 mo $n(\%)$ & $p \mathrm{~b}$ \\
\hline Hemorrhage & $54(43.9)$ & $20(16.3)$ & $<0.0001$ & $10(8.1)$ & 0.0782 \\
RPE atrophy & $13(10.6)$ & $32(26.0)$ & 0.0027 & $45(35.6)$ & 0.0986 \\
Fibrosis & $3(2.4)$ & $9(7.3)$ & 0.1361 & $16(13.0)$ & 0.2048 \\
Dry macula & $12(9.8)$ & $76(61.8)$ & $<0.0001$ & $83(67.5)$ & $<0.0001$ \\
\hline
\end{tabular}

RPE: retinal pigment epithelium; LD: loading dose; 12 mo: final follow-up; $n(\%)$, number of eyes (percentage), pa: differences baseline vs. LD; $p$ b: differences LD vs. 12 mo; and $p$ c: differences baseline vs. 12 mo.

of 4.9 letters, this improvement was not statistically significant, perhaps due to the reduced size of this population or due to a more often central macular affectation. It should be noted that as per our standard protocol in a daily clinical practice, RAP or PCV cases were not diagnosed by FA or ICGA at baseline, as these imaging techniques are only used for some patients who have not responded to treatment.

The mean age of the patients is among the highest compared to all previous studies with aflibercept. Unexpectedly, BCVA at the time of diagnosis was also among the highest, which likely reflects the effort made in our setting over the last years to speed referral from primary care to ophthalmology offices. Both higher age and higher BCVA at baseline are known to be associated with "ceiling effect" and then linked to lower VA gains $[11,18,24]$. Despite these differences, other factors with a clear effect on visual outcomes should be considered when interpreting these results. These include factors associated with early detection of AMD and access to treatment, as well as whether the outcomes reported include fellow eyes when both eyes are affected. Our study has also shown that the percentage of eyes that had some degree of RPE atrophy before treatment was significantly higher in the subgroup of older patients. In any case, long-term data indicate that despite an initial good response, VA inexorably deteriorates given the progressive nature of the disease $[11,25]$.

Mean VA gain has been used as an outcome measure to assess efficacy in noninferiority clinical trials; however, more patient-centred measures of success may be preferred in studies in the real-world, including the percentage of patients who did not lose vision, or who gained $\geq 15$ letters or maintained/achieved functional vision (i.e., $\geq 20 / 40$ or 70 letters). After 12 -month treatment, $88.6 \%$ of the patients in our study had not lost vision (i.e., had not lost $\geq 10$ letters), $18.7 \%$ had gained $\geq 15$ letters, and the percentage of patients maintaining or achieving a BCVA $\geq 70$ letters had increased from $30.1 \%$ at baseline to $46.3 \%$. As with the mean VA gain, these figures were slightly worse than those reported in pivotal studies [13].

Our study also reflects significant improvements in anatomic outcomes. As expected, there was a significant reduction in $\mathrm{CMT}$ after the $\mathrm{LD}$, remaining stable up to month 12. Mean change in CMT and AMT at month 12 was lower than that reported in pivotal clinical trials [13] but similar to that reported in other real-world studies $[10,15,20,26]$. Despite that, the percentage of eyes with dry macula at final visit was similar to that reported previously $[13,15,26]$.

Compliance with treatment and regular visits has also been shown to influence effectiveness of anti-VEGF therapy in the real world $[17,27]$. This aspect is especially relevant among the elderly as it may be influenced by a number of everyday reasons (falls, concurrent illnesses, etc.) [18]. Our study includes all consecutive treated patients for whom 12month treatment information was available. Patients received a mean of 6.8 IVA, with $9 \%$ of patients receiving 8 


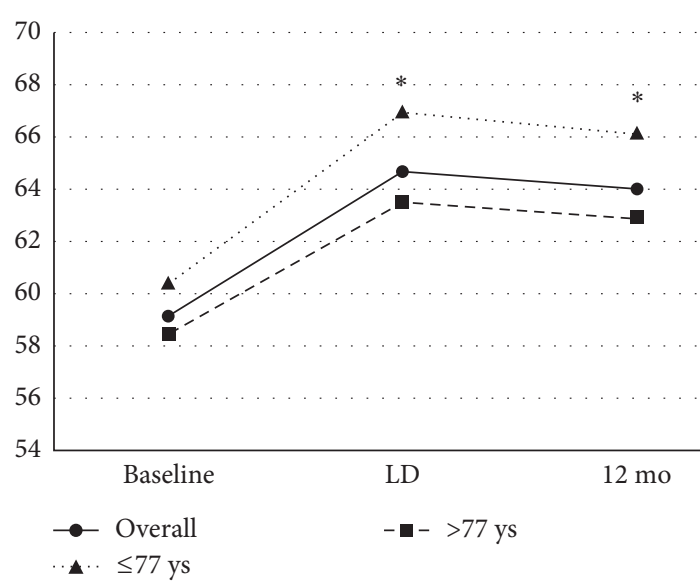

(a)

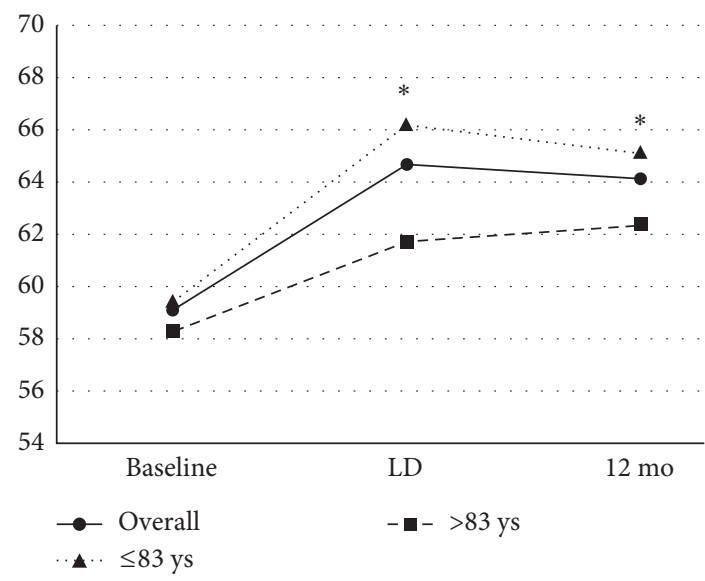

(c)

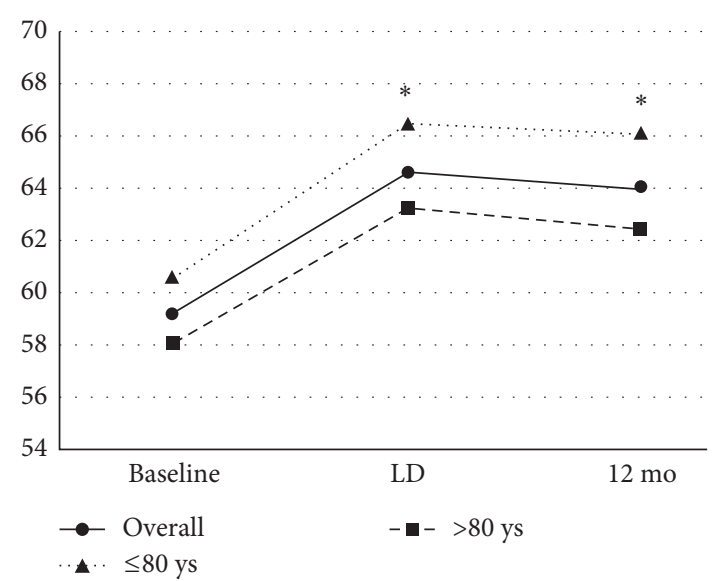

(b)

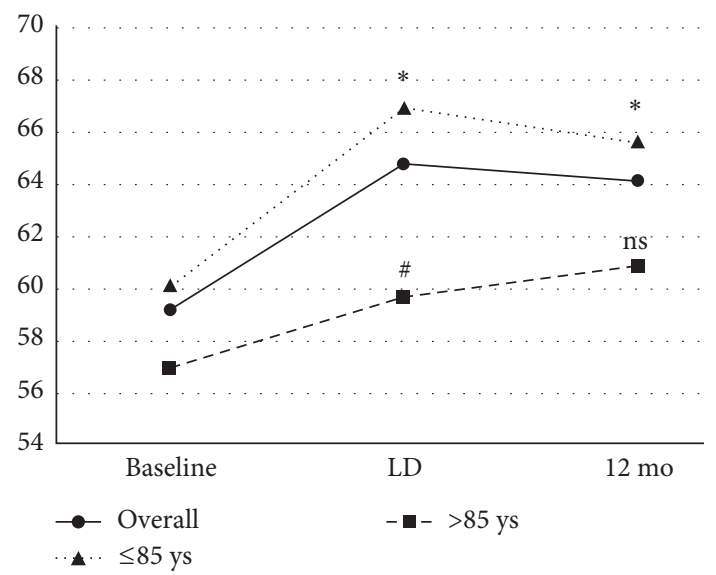

(d)

Figure 3: Changes in BCVA (ETDRS letters) by age subgroup. (a) Patients $\leq 77$ vs. $>77$ years. (b) Patients $\leq 80$ vs. $>80$ years. (c) Patients $\leq 83$ vs. $>83$ years. (d) Patients $\leq 85$ vs. $>85$ years. Mean changes in BCVA after the loading dose (LD) and at the final follow-up were significant $\left({ }^{*}\right)$. The eyes belonging to the older subgroups (>85 years old), although they presented significant (\#) gains in the BCVA means after the LD, there were not significant differences at 12 months $(12 \mathrm{mo})$. ns: not significant.

TABLE 3: Best-corrected visual acuity ( $\mathrm{MD} \pm \mathrm{SD}$, number of letters) and gain (number of letters) at the baselines and at final follow-up period (12 mo) by age subgroup.

\begin{tabular}{lcccc}
\hline Age subgroup & Baseline & $12 \mathrm{mo}$ & Gain & $p$ \\
\hline$\leq 77 \mathrm{y}(n=43)$ & $60.4 \pm 18.5$ & $66.1 \pm 16.1$ & 5.7 & 0.02 \\
$\leq 80 \mathrm{y}(n=59)$ & $60.3 \pm 17.3$ & $66.1 \pm 15.0$ & 5.8 & 0.009 \\
$\leq 83 \mathrm{y}(n=79)$ & $59.5 \pm 18.3$ & $65.1 \pm 15.0$ & 5.6 & 0.001 \\
$\leq 85 \mathrm{y}(n=89)$ & $60.0 \pm 17.6$ & $65.5 \pm 14.7$ & 5.5 & 0.008 \\
$>77 \mathrm{y}(n=80)$ & $58.4 \pm 15.3$ & $62.9 \pm 13.4$ & 4.5 & 0.004 \\
$>80 \mathrm{y}(n=64)$ & $57.9 \pm 15.8$ & $62.5 \pm 13.8$ & 4.6 & 0.008 \\
$>83 \mathrm{y}(n=44)$ & $58.3 \pm 12.6$ & $62.3 \pm 13.2$ & 4.0 & 0.02 \\
$>85 \mathrm{y}(n=34)$ & $56.9 \pm 12.6$ & $60.7 \pm 13.2$ & 3.8 & 0.07 \\
\hline
\end{tabular}

$y$ : age in years; $n$ : number of eyes; 12 mo: final follow-up; and gain: BCVA in number of letters ETDRS.

injections and 34\% receiving 6 injections within the year instead of the expected 7, which was likely to be due to administration adjustments and the referred patients.

Our standard operating procedure for intravitreal treatment has been able to substantially reduce the number of follow-up visits (average 3 visits/year). Taking into account both the regular compliance with intravitreal treatment and the considerable decrease in healthcare pressure with fewer follow-up visits, treatment guidelines with a fixed regimen represent important advantages for the public healthcare system. Furthermore, our results have been able to demonstrate that the treatment based on a fixed regimen is capable of maintaining a high percentage of eyes with stable VA and anatomic profiles of the macula without IRF or SRF.

Despite that, our study has also several limitations, mostly related to the relatively small sample size and its retrospective nature, especially with regard to variables or confounding factors not collected (i.e., time from first symptoms to diagnosis and from diagnosis to treatment) that could have better explained the results observed. As previously remarked, potential PCV and RAP cases were not diagnosed at baseline, and therefore the differential outcomes according to type of nAMD are based on signs pointing to these nAMD variants. The effect of fellow eyes was not analyzed, which affected 17 eyes. The main strength of our analysis is that it was conducted in a single centre, with satisfactory level of compliance under a standardized 


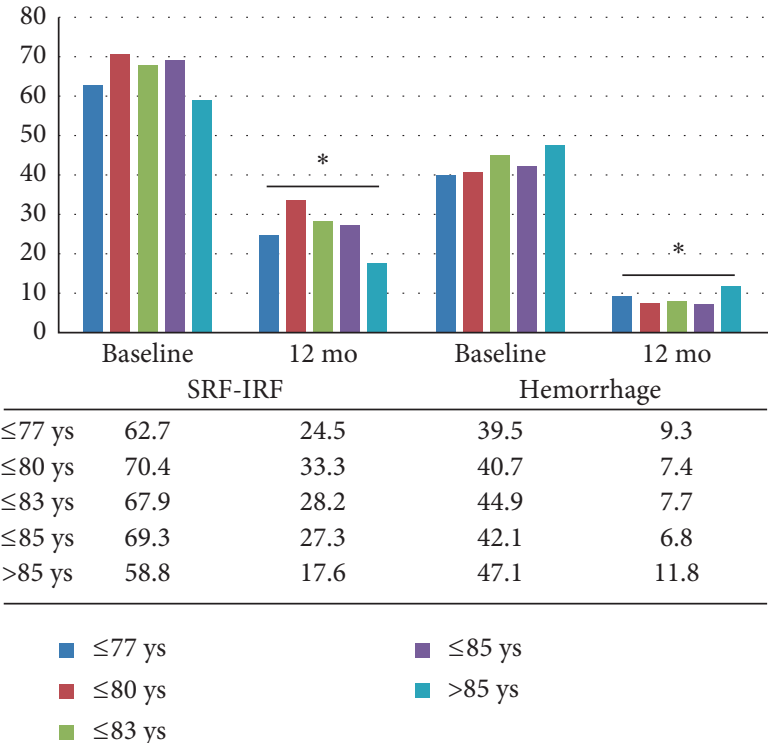

(a)

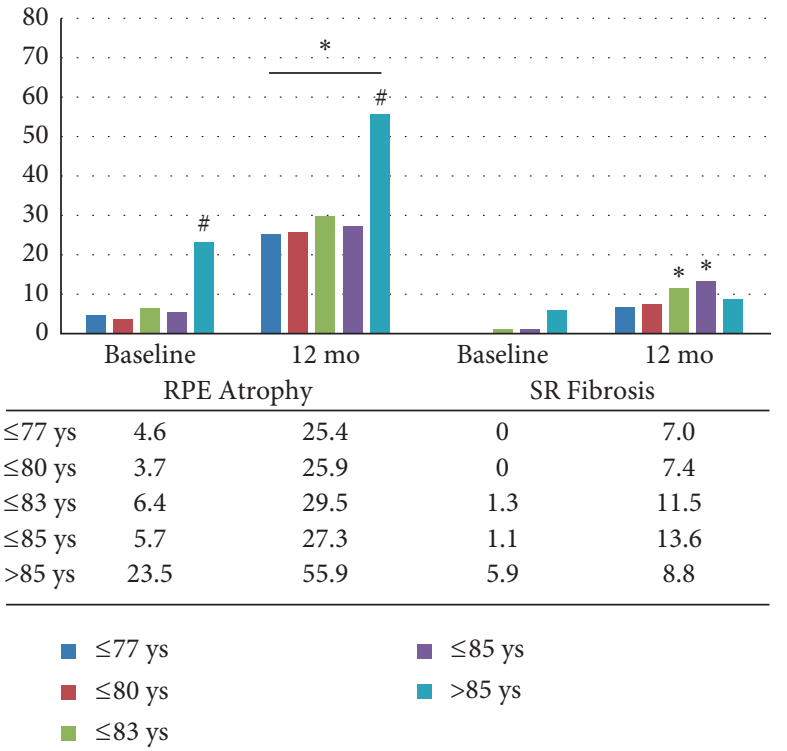

(b)

FIgURE 4: Presence of retinal findings during the period of study by age subgroups. (a) Changes in percentages of eyes with subretinal fluid (SFR), intraretinal fluid (IRF), and hemorrhages and (b) percentage of eyes presenting retinal pigment epithelium (RPE) atrophy and subretinal (SR) fibrosis, at baseline, after the loading dose (LD) and at 12 months (12 mo), were significant $\left({ }^{*}\right)$. Percentage of eyes in the subgroup of patients with more than 85 years presented significant (\#) RPE atrophy at the baseline and at final follow-up.

protocol, thus ensuring the homogeneity of patient management.

\section{Conclusions}

The results of our study support the visual and anatomic benefits of fixed regimen of aflibercept in real-life clinical practice in naïve patients with nAMD. Significant improvements were achieved after the LD and maintained up to one year. Compliance with the bimonthly dosing and safety profile were generally satisfactory. These improvements are in agreement with those reported in other studies in clinical practice and support our management therapeutic protocol and logistic in public health specialized care.

\section{Data Availability}

The data used to support the findings of this study are available from the corresponding author upon request.

\section{Conflicts of Interest}

The authors declare that they have no proprietary interest regarding the publication of this paper.

\section{Acknowledgments}

The authors thank Beatriz Viejo, Ph.D., for her assistance in the writing of the manuscript and David Calbet for his statistical support. The authors also thank all optometric team members of their ophthalmological service for helping them in the clinical practice results observed in this study.

\section{References}

[1] N. M. Bressler, "Age-related macular degeneration is the leading cause of blindness," JAMA, vol. 291, no. 15, pp. 1900-1901, 2004.

[2] N. G. Congdon, D. S. Friedman, and T. Lietman, "Important causes of visual impairment in the world today," JAMA, vol. 290, no. 15, pp. 2057-2060, 2003.

[3] P. Mitchell, G. Liew, B. Gopinath, and T. Y. Wong, "Agerelated macular degeneration," The Lancet, vol. 392, no. 10153, pp. 1147-1159, 2018.

[4] C. L. Nguyen, M. C. Gillies, V. Nguyen et al., "Characterization of poor visual outcomes of neovascular age-related macular degeneration treated with anti-vascular endothelial growth factor Agents," Ophthalmology, vol. 126, no. 5, pp. 735-742, 2019.

[5] A. R. Rudnicka, Z. Jarrar, R. Wormald, D. G. Cook, A. Fletcher, and C. G. Owen, "Age and gender variations in age-related macular degeneration prevalence in populations of European ancestry: a meta-analysis," Ophthalmology, vol. 119, no. 3, pp. 571-580, 2012.

[6] A. Garcia-Layana, F. Cabrera-López, J. García-Arumí, L. Arias-Barquet, and J. M. Ruiz-Moreno, "Early and intermediate age-related macular degeneration: update and clinical review," Clinical Interventions in Aging, vol. 12, pp. 15791587, 2017.

[7] J. Q. Li, T. Welchowski, M. Schmid, M. M. Mauschitz, F. G. Holz, and R. P. Finger, "Prevalence and incidence of agerelated macular degeneration in Europe: a systematic review and meta-analysis," British Journal of Ophthalmology, vol. 104, no. 8, pp. 1077-1084, 2020.

[8] U. Schmidt-Erfurth, V. Chong, A. Loewenstein et al., "Guidelines for the management of neovascular age-related macular degeneration by the European Society of Retina 
Specialists (EURETINA)," British Journal of Ophthalmology, vol. 98, no. 9, pp. 1144-1167, 2014.

[9] A. Invernizzi, V. Nguyen, K. Teo et al., "Five-year real-world outcomes of occult and classic choroidal neovascularization: data from the Fight Retinal Blindness! Project," American Journal of Ophthalmology, vol. 204, pp. 105-112, 2019.

[10] J. Carrasco, G.-A. Pietsch, M.-P. Nicolas, C. Koerber, C. Bennison, and J. Yoon, "Real-world effectiveness and realworld cost-effectiveness of intravitreal aflibercept and intravitreal ranibizumab in neovascular age-related macular degeneration: systematic review and meta-analysis of real-world studies," Advances in Therapy, vol. 37, no. 1, pp. 300-315, 2020.

[11] F. G. Holz, M. S. Figueroa, F. Bandello et al., "Ranibizumab treatment in treatment-naive neovascular age-related macular degeneration: results from luminous, a global real-world study," Retina, vol. 40, no. 9, pp. 1673-1685, 2019.

[12] M. W. Stewart, P. J. Rosenfeld, F. M. Penha et al., "Pharmacokinetic rationale for dosing every 2 weeks versus 4 weeks with intravitreal ranibizumab, bevacizumab, and aflibercept (vascular endothelial growth factor Trap-eye)," Retina (Philadelphia, PA, USA), vol. 32, no. 3, pp. 434-457, 2012.

[13] J. S. Heier, D. M. Brown, V. Chong et al., "Intravitreal aflibercept (VEGF trap-eye) in wet age-related macular degeneration," Ophthalmology, vol. 119, no. 12, pp. 2537-2548, 2012.

[14] M. Eleftheriadou, M. Gemenetzi, M. Lukic et al., "Three-year outcomes of aflibercept treatment for neovascular age-related macular degeneration: evidence from a clinical setting," Ophthalmology and Therapy, vol. 7, no. 2, pp. 361-368, 2018.

[15] H. Almuhtaseb, S. Kanavati, S. R. Rufai, and A. J. Lotery, "One-year real-world outcomes in patients receiving fixeddosing aflibercept for neovascular age-related macular degeneration," Eye, vol. 31, no. 6, pp. 878-883, 2017.

[16] P. Udaondo, D. Salom, S. García-Delpech, and Á. CisnerosLanuza, "Aflibercept as first-line therapy in patients with treatment-naïve neovascular age-related macular degeneration: prospective case series analysis in real-life clinical practice," Ophthalmologica, vol. 236, no. 1, pp. 29-35, 2016.

[17] C. Framme, N. Eter, T. Hamacher et al., "Aflibercept for patients with neovascular age-related macular degeneration in routine clinical practice in Germany," Ophthalmology Retina, vol. 2, no. 6, pp. 539-549, 2018.

[18] J. S. Talks, A. J. Lotery, F. Ghanchi et al., "First-year visual acuity outcomes of providing aflibercept according to the VIEW study protocol for age-related macular degeneration," Ophthalmology, vol. 123, no. 2, pp. 337-343, 2016.

[19] K. Nishikawa, A. Oishi, M. Hata et al., "Four-year outcome of aflibercept for neovascular age-related macular degeneration and polypoidal choroidal vasculopathy," Scientific Reports, vol. 9, no. 1, p. 3620, 2019.

[20] M. Y. Guo, J. Cheng, M. Etminan, Z. Zafari, and D. Maberley, "One year effectiveness study of intravitreal aflibercept in neovascular age-related macular degeneration: a meta-analysis," Acta Ophthalmologica, vol. 97, no. 1, pp. e1-e7, 2019.

[21] P. Tewarie, L. Balk, F. Costello et al., "The OSCAR-IB consensus criteria for retinal OCT quality assessment," PLoS One, vol. 7, Article ID e34823, 2012.

[22] C. M. G. Cheung, T. Y. Y. Lai, P. Ruamviboonsuk et al., "Polypoidal choroidal vasculopathy," Ophthalmology, vol. 125 , no. 5 , pp. 708-724, 2018.

[23] A. S. H. Tsai, N. Cheung, A. T. L. Gan et al., "Retinal angiomatous proliferation," Survey of Ophthalmology, vol. 62, no. 4, pp. 462-492, 2017.
[24] G.-s. Ying, J. Huang, M. G. Maguire et al., "Baseline predictors for one-year visual outcomes with ranibizumab or bevacizumab for neovascular age-related macular degeneration," Ophthalmology, vol. 120, no. 1, pp. 122-129, 2013.

[25] S. Rofagha, R. B. Bhisitkul, D. S. Boyer, S. R. Sadda, and K. Zhang, "Seven-year outcomes in ranibizumab-treated patients in ANCHOR, MARINA, and HORIZON," Ophthalmology, vol. 120, no. 11, pp. 2292-2299, 2013.

[26] M. Eleftheriadou, C. Vazquez-Alfageme, C. M. Citu et al., "Long-term outcomes of aflibercept treatment for neovascular age-related macular degeneration in a clinical setting," American Journal of Ophthalmology, vol. 174, pp. 160-168, 2017.

[27] F. G. Holz, R. Tadayoni, S. Beatty et al., "Multi-country reallife experience of anti-vascular endothelial growth factor therapy for wet age-related macular degeneration," British Journal of Ophthalmology, vol. 99, no. 2, pp. 220-226, 2015. 